Draft Version November 21, 2018

Preprint typeset using $\mathrm{L}^{A} \mathrm{~T}_{\mathrm{E}} \mathrm{X}$ style emulateapj v. 11/10/09

\title{
INTEGRATED STELLAR POPULATIONS: CONFRONTING PHOTOMETRY WITH SPECTROSCOPY
}

\author{
Lauren A. MacArthur ${ }^{1,2}$, Michael McDonald ${ }^{3}$, Stéphane Courteau $^{4}$, and J. Jesús GonzÁlez ${ }^{5}$ \\ Draft version November 21, 2018
}

\begin{abstract}
We investigate the ability of spectroscopic techniques to yield realistic star formation histories (SFHs) for the bulges of spiral galaxies based on a comparison with their observed broadband colors. Full spectrum fitting to optical spectra indicates that recent (within $\sim 1 \mathrm{Gyr}$ ) star formation activity can contribute significantly to the $V$-band flux, whilst accounting for only a minor fraction of the stellar mass budget which is made up primarily of old stars. Furthermore, recent implementations of stellar population (SP) models reveal that the inclusion of a more complete treatment of the thermally pulsating asymptotic giant branch (TP-AGB) phase to SP models greatly increases the NIR flux for SPs of ages 0.2-2 Gyr. Comparing the optical-NIR colors predicted from population synthesis fitting, using models which do not include all stages of the TP-AGB phase, to the observed colors reveals that observed optical-NIR colors are too red compared to the model predictions. However, when a 1 Gyr SP from models including a full treatment the TP-AGB phase is used, the observed and predicted colors are in good agreement. This has strong implications for the interpretation of stellar populations, dust content, and SFHs derived from colors alone.
\end{abstract}

Subject headings: galaxies: bulges — galaxies: evolution — galaxies: formation — galaxies: stellar content

\section{INTRODUCTION}

A detailed understanding of the stellar populations (SPs) that make up the integrated spectral energy distributions (SEDs) of both local and distant galaxies can provide important constraints for models of galaxy formation. However, confronting observations with SP synthesis models for the purpose of translating the former into physical parameters, is not without significant challenges. While SP modeling has seen tremendous progress over the past decade (e.g., Bruzual \& Charlot 2003, hereafter BC03; Le Borgne et al. 2004; Maraston 2005, hereafter Mar05; Schiavon 2007), misinterpretations in the data-model comparison are still common.

Broadband colors are often used as a proxy for SP parameters. The well-known age/ $Z$ degeneracy in optical colors is greatly lifted by the addition of NIR bands. However, in broadband-based analyses, extinction and reddening effects from interstellar dust cannot be ruled out. Indeed, observed optical-NIR colors that lie redward of the model grids, i.e., in a region not supported by a naked SP of any age/ $Z / \mathrm{SFH}$, have typically been attributed to dust reddening (e.g., Peletier et al. 1999; MacArthur et al. 2004; Carollo et al. 2007). Meanwhile, red optical-NIR colors within the model grids are assumed to result from old and metal-rich SPs.

In light of recent implementations of SP models which account for a more detailed treatment of the effects of

\footnotetext{
${ }^{1}$ Herzberg Institute of Astrophysics, National Research Council of Canada, 5071 West Saanich Road, Victoria, BC V8X 4M6, Canada; Lauren.MacArthur@nrc-cnrc.gc.ca

2 Department of Physics \& Astronomy, University of Victoria, Victoria, BC, V8P 1A1, Canada

3 Department of Astronomy, University of Maryland, College Park, MD 20742-2421, USA

${ }^{4}$ Department of Physics, Engineering Physics \& Astronomy, Queen's University, Kingston, ON K7L 3N6, Canada

${ }^{5}$ Instituto de Astronomia, Universidad Nacional Autónoma de México, Apdo Postal 70-264, Cd. Universitaria, 04510, Mexico
}

the thermally pulsating asymptotic giant branch (TPAGB) stellar evolutionary phase (e.g., Mar05; Coelho et al. 2007; Lee et al. 2009), a reassessment of the observed red optical-NIR colors in galaxies is in order. Accounting for the TP-AGB is accomplished in Mar05 through empirical calibration to LMC globular clusters which have independent age and $Z$ measurements. Alternatively, synthetic model tracks can be used. The recent tracks of Marigo \& Girardi (2007), whose predictions are in good agreement with those of Mar05, account for nine evolutionary stages (including the C-, M-type, and superwind mass loss phases) in the TP-AGB. (Note that the older tracks used in the BC03 models account for only a single evolutionary stage for each evolutionary phase in the TP-AGB.) In these models, the TP-AGB phase is active in SPs of ages $\sim 0.3-2 \mathrm{Gyr}$ and contributes significantly to the NIR flux (accounting for up to $\sim 80 \%$ of the NIR flux), leading to very red optical-NIR colors for this age range. These models have already been used to resolve the uncomfortably large stellar masses and old ages derived for high- $z$ galaxies using models which do not account for all phases in their treatment of the TPAGB phase (Maraston et al. 2006).

In the meantime, full spectrum fitting techniques have proven to be effective at recovering the underlying stellar content of integrated galaxy spectra (e.g., Heavens et al. 2000; Cid Fernandes et al. 2005; Walcher et al. 2006: MacArthur, González, \& Courteau 2009, hereafter Mac09). This type of analysis provides a stochasticallysampled SFH for a given integrated spectrum and allows for true average, as opposed to single SP-based, age and $Z$ estimates. Full spectral fitting is typically limited to optical wavelengths, as set by the observations. However, as most models provide SEDs in the UV-FIR range, one can use such model fits to make predictions for the galaxy light in other wavelength regions. For example, a comparison of predicted versus observed optical-NIR colors could provide valuable insight into both the reliability 
of the spectral fits as well as any shortcomings of the SP models themselves. This is precisely the approach we carry out here using our analysis of Gemini/GMOS long-slit spectra of local spirals (Mac09) and new optical imaging for the same galaxies from the Palomar Observatory and NIR imaging from the Two Micron All Sky Survey (hereafter 2MASS; Skrutskie et al. 2006). As we are interested in predictions from models that use different treatments of the TP-AGB phase, we focus on two galaxies from our spectroscopic sample (NGC 628 \& UGC 2124) with the highest quality data and whose derived SFHs indicate significant contribution (in $V$-band light-weight) from a 1 Gyr SP, i.e., where the TP-AGB NIR signature is expected to be strong.

\section{DATA}

The long-slit spectroscopic data used for this study, from Mac09, were collected with the Gemini MultiObject Spectrograph (GMOS; Hook et al. 2004) on the 8-m Gemini North telescope. The GMOS detector and B600 grating combination provides a spatial resolution of $0.072^{\prime \prime} / \mathrm{pix}$ and a dispersion of $0.45 \AA /$ pix. The slit field of view (FOV) of $5^{\prime} \times 2^{\prime \prime}$ provided a boxcar width $=10.81 \AA$ resolution. The spectral coverage spans $\sim 4050-6750 \AA$.

Optical imaging in the BVRI Bessel filters was collected at the Palomar 200-inch telescope in 2006 December. The Large Format Camera consists of a six CCD mosaic with a FOV of $6 \times 12^{\prime}$ per CCD. Our galaxies have optical diameters $\leq 10.5^{\prime}$, leaving enough room beyond the optical radius on a single chip for sky measurements. Typical exposure times were 1-2 minutes and $2 \times 2$ binning gave $0.363^{\prime \prime} /$ pixel. The seeing ranged from $\mathrm{FWHM}=1.8-3.7^{\prime \prime}$. The data were bias-subtracted and flat-fielded using a combination of night-sky and dome flats to carefully account for both the high-frequency spatial sensitivity (dome) and the large-scale illumination (sky) of the chip. Due to the bright-moon conditions, it was necessary to model the background with a surface. This induced a typical sky error of $\sim 0.01 \mathrm{mag}$. The data were calibrated following the technique of Courteau (1996) using stars from several Landolt fields (Landolt 1992), for typical calibration errors of $\sim 0.03 \mathrm{mag}$.

Imaging in the NIR JHKs filters were obtained from the 2MASS online database. The background levels were carefully re-measured for each image and the photometric zeropoints were taken from the 2MASS headers.

All data (imaging and spectroscopic) were corrected for Galactic foreground extinction (Schlegel, Finkbeiner, \& Davis 1998).

\subsection{Radial Extractions}

In order to make a direct comparison between information gleaned from optical spectra versus broadband imaging, we must compare the spectral fits with colors from the same effective radial bins. As such, rather than the usual azimuthal extraction of SB profiles (e.g.,, Courteau 1996; MacArthur, Courteau, \& Holtzman 2003), profiles were extracted from all bands $(B V R I J H K s)$ using the same position angle and slit width as for the GMOS observations. We accounted for differences in the observed point spread functions by convolving all profiles (imaging and spectroscopic) with the largest FWHM of all obser- vations for each galaxy. Finally, radial binning for both the imaging and spectroscopy was set to the largest measured dispersion, which is that of 2MASS $\left(1^{\prime \prime} /\right.$ pixel).

\section{AGE, METALLICITY, AND SFH FROM POPULATION SYNTHESIS}

The SP fitting technique used here is as described in Mac09; a brief summary is provided below. Our "full population synthesis" technique consists of a bound constrained optimized fit representing the relative contribution of each of the 70 model templates to the observed spectrum. The only fixed bound is that of no negative template contributions. The templates are from the BC03 models which provide SEDs representing simple stellar populations (SSPs), i.e., single bursts of star formation (SF) at a given age and $Z$, at a resolution of $\sim 3 \AA$ FWHM covers the age range $0.001-20 \mathrm{Gyr}$ and metallicities of $Z=0.0004-0.05$ and we use the models with the Chabrier (2003) initial mass function. Our procedure also allows for dust reddening to the observed SED according to the prescription of Charlot \& Fall (2000). See Mac09 for details. To accommodate any non-stellar contributions to the observed spectrum not considered in the $\mathrm{BC} 03$ models, we used an iterative masking scheme whereby deviant |data-model| points are given zero weight in the fit.

Figure 1 shows the full population synthesis fits to the central spectra of NGC 628 and UGC 2124 (extracted as described in Section 2.11), and in Figure 2 we plot the inferred SFHs from the fits. In Table 1, we list for each galaxy the percent-light and mass contributions of "very young" (0.001-0.4 Gyr), "young" (1-2 Gyr), "intermediate" (4-7 Gyr), and "old" (10-20 Gyr) age SSPs to the fits.

For both galaxies there is a contribution from 13 to 20 Gyr SSP 4 . While their weight in light may not seem dominant, these old populations contribute $74 \%$ and $93 \%$ to the stellar mass budget of NGC 628 and UGC 2124, respectively (Table 1). However, we are particularly interested here in a significant contribution from SSPs that would be expected by the Mar05 models to have NIR excesses from their treatment of the TP-AGB stellar evolution phase. The age range for which the signature is present is highlighted by the gray shading in Figure 2 and referred to as "young" in Table 1. The contribution to the $V$-band normalized light of the young population of interest is $67 \%$ and $33 \%$ for NGC 628 and UGC 2124, respectively. Thus, if our SFHs are reliable, according to the Mar05 models, these galaxies would be expected to show signs of the TP-AGB NIR exces: 8 .

\section{4. "PREDICTED" VERSUS OBSERVED OPTICAL-NIR COLORS}

\footnotetext{
${ }^{6}$ Although see $\S 3.4 .1$ of Mac09 for a detailed analysis of the true resolution of these models.

7 We are not concerned here with model ages that are older than the age of the universe as absolute model SSP ages are not precisely calibrated.

8 Note that we did not perform the population synthesis fits with the Mar05 models as they have a lower resolution than $\mathrm{BC} 03$, thus reducing the predictive power of the spectra to disentangle the high frequency features in the fitting process. A direct comparison between fits using different models awaits the release of higher resolution models.
} 

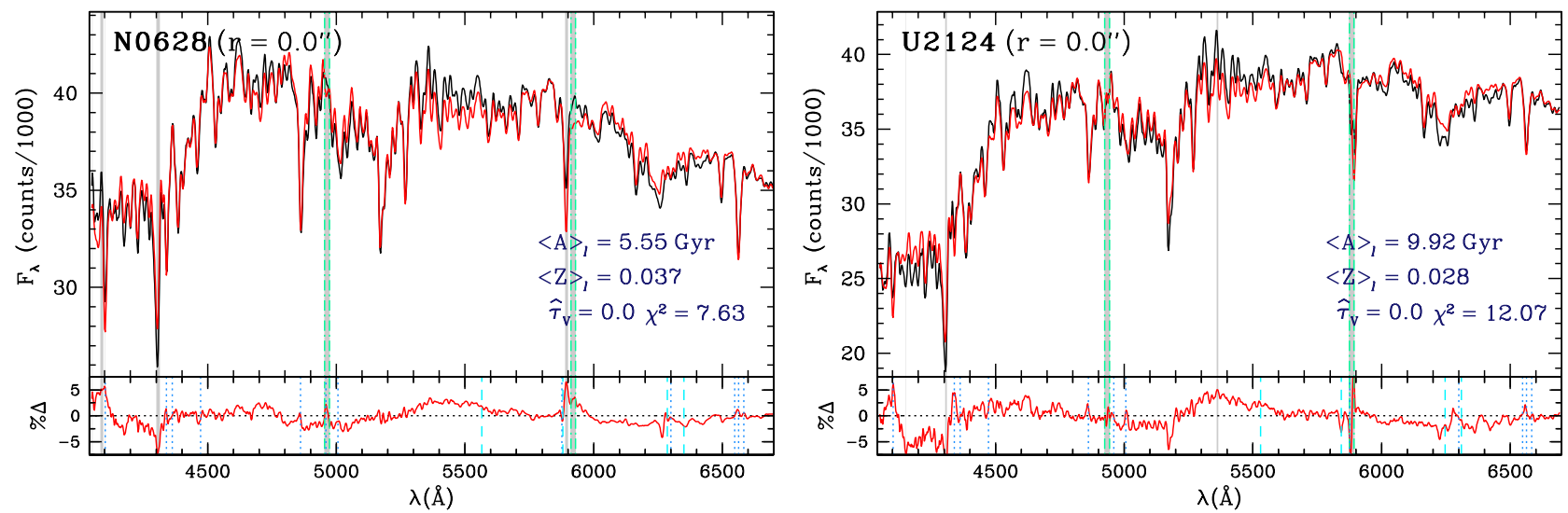

FIG. 1.- Central observed spectra (black) and full population synthesis fit (red) for NGC 628 (left) and UGC 2124 (right). Gray shading indicates regions masked in the fit as determined by our iterative " $\sigma$-clipping" procedure as well as the CCD gap regions (green vertical dash-dotted lines) which are always masked. Shown at lower right on each panel are the average light-weighted age, $\langle\mathrm{A}\rangle_{l}$, metallicity, $\langle\mathrm{Z}\rangle_{l}$ , effective $\hat{\tau}_{V}$, and $\chi^{2}$ of the fit. The bottom panels show the percent data-model residuals. In the bottom panels, dashed vertical lines indicate variable sky-lines, and dotted vertical lines indicate emission lines prevalent in star forming (H II) regions.

TABLE 1

Percent Contributions of all SSPs in Given Age Ranges (Very Young $=0.001-0.4$ Gyr; Young $=1-2$ Gyr; Intermediate $=4-7$ Gyr; Old $=10-20$ Gyr) to Full Population Synthesis Fits Weighted by Light $(V$-Band normalized) and MASS.

\begin{tabular}{|c|c|c|c|c|c|c|c|c|}
\hline \multirow[b]{2}{*}{ Name } & \multicolumn{4}{|c|}{ Light Weight } & \multicolumn{4}{|c|}{ Mass Weight } \\
\hline & $\begin{array}{c}0.001-0.4 \\
(\mathrm{Gyr})\end{array}$ & $\begin{array}{c}1-2 \\
(\mathrm{Gyr})\end{array}$ & $\begin{array}{c}4-7 \\
(\mathrm{Gyr}) \\
\end{array}$ & $\begin{array}{l}10-20 \\
\text { (Gyr) }\end{array}$ & $\begin{array}{c}0.001-0.4 \\
\quad(\mathrm{Gyr})\end{array}$ & $\begin{array}{c}1-2 \\
(\mathrm{Gyr})\end{array}$ & $\begin{array}{c}4-7 \\
(\mathrm{Gyr})\end{array}$ & $\begin{array}{l}10-20 \\
\text { (Gyr) }\end{array}$ \\
\hline N0628 & 7 & 67 & 0 & 26 & 1 & 25 & 0 & 74 \\
\hline $\mathrm{U} 2124$ & 0 & 33 & 0 & 67 & 0 & 7 & 0 & 93 \\
\hline
\end{tabular}

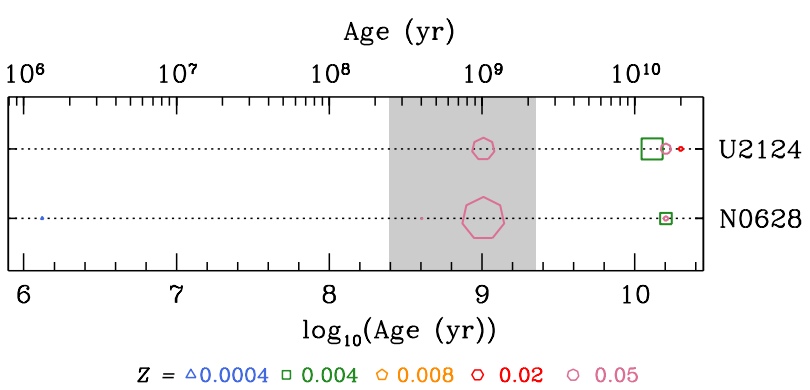

FIG. 2.- Light-weighted SFHs of the central spectra for NGC 628 and UGC 2124 derived from the full population synthesis fits. The dotted horizontal lines guide the eye for each galaxy (labeled at right). The horizontal axis is the SSP age. Point size is proportional to the relative weight in the fit (normalized to the $V$-band) and the colors and point types code the SSP metallicity, as indicated. The gray shading indicates the age range within which the TP-AGB phase is active.

The full population synthesis fits to the spectra are constrained by the observed $4050-6750 \AA$ region (gray shading in Figure 31) and, modulo emission lines, represent this region very well (see Figure 1). Since the BC03 models cover the range $90 \AA-160 \mu \mathrm{m}$, we can use the model fits to "predict" colors in other bands. Any difference between colors predicted in this fashion and the observed colors can be explained by any of the following scenarios: unrealistic SFHs due to degeneracies in the age $/ Z /$ dust plane that cannot be constrained with op- tical data alone; inadequacy of the template library to represent the full coverage of SP age and $Z$ in any given integrated spectrum; unrealistic estimate of the dust extinction from the population synthesis fits; errors in the SP modeling predictions outside the optical range due to, for example, errors in the stellar evolutionary physics.

To test for any discrepancies, we now compare the predicted optical-NIR colors from the full population synthesis BC03 model fits to the observed colors. A direct estimate of the predicted NIR excess is complicated by a number of issues related to differences between the BC03 and Mar05 models. First, for a given age and $Z$, the model predictions are not identical, even within the optical limits of our spectra. Second, the SEDs provided in each model do not cover precisely the same age $/ Z$ grid. To illustrate differences between the $\mathrm{BC} 03$ and Mar05 model SEDs, we compare in Figure 3 the $1 \mathrm{Gyr} / Z=0.05$ SSP from BC03, which contributes significantly to both SFHs in Figure 2, to the SED closest in age and $Z$ from the Mar05 models $(1 \mathrm{Gyr} / Z=0.04)$. While most of the difference is attributed to the different treatments of the TP-AGB phase between $\mathrm{BC} 03$ and Mar05, some of it could also be due to the overall larger contribution of AGB stars in the Mar05 models. For this comparison, the BC03 models were smoothed with a boxcar of $\mathrm{FWHM}=20 \AA$ and resampled to $\Delta \lambda=20 \AA /$ pix to roughly match the resolution and sampling of the Mar05 SEDs.

Given the above caveats, we now look at the observed versus predicted optical-NIR colors for NGC 628 in Fig- 


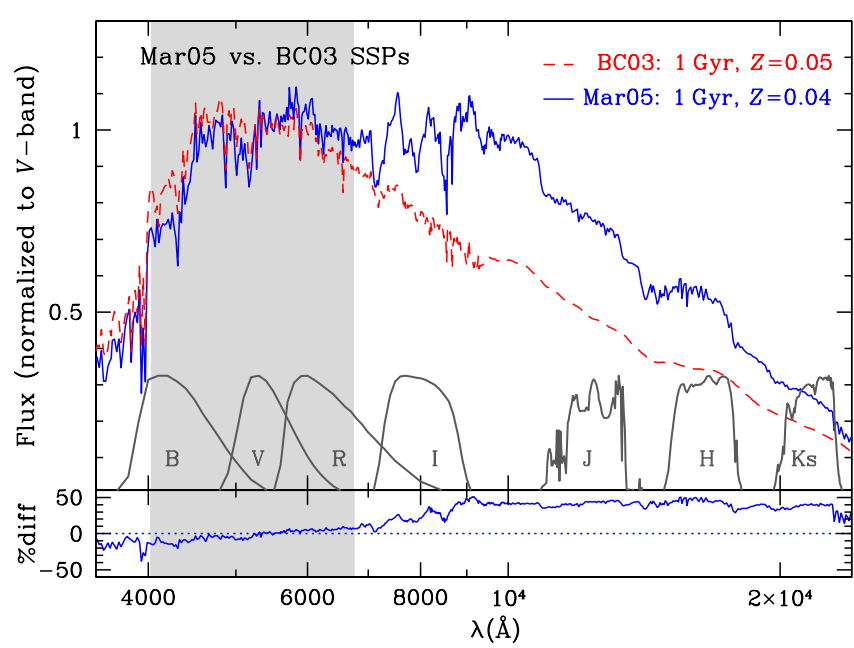

FIG. 3.- Comparison of SSP SEDs for the Mar05 and BC03 models. The dashed red line is the $1 \mathrm{Gyr} / Z=0.05 \mathrm{SSP}$ from the BC03 models. The solid blue line is the $1 \mathrm{Gyr} / Z=0.04$ SSP from the Mar05 models. The relevant filter response curves for our observed colors are shown as dark gray curves in the upper panel. The lower panel plots the model differences, where $\%$ diff $=100 *(\mathrm{BC} 03-\mathrm{Mar05}) /[(\mathrm{BC} 03+\mathrm{Mar} 05) / 2]$. The gray shading represents the optical range of our GMOS spectra within which the population fits are constrained.

ure 4. In all color combinations, the model fits (red open triangles) predict colors that are too blue compared to the observed (black solid triangles) optical-NIR colors. The blue arrows represent the difference in color between the BC03 $1 \mathrm{Gyr} / Z=0.05 \mathrm{SSP}$ and the Mar05 $1 \mathrm{Gyr} / Z=0.04 \mathrm{SSP}$. Indeed, the blue vectors represent the data-model difference very well. For UGC 2124 in Figure [5, it appears that a steeper vector would be required to precisely match the data-model difference. In Mac09 we demonstrated by a comparison of absorptionline indices that there is evidence of an enhanced $[\alpha / \mathrm{Fe}]$ $\mathrm{SP}$ in the bulge region of UGC 2124 (see Figure 4 in Mac09), which could also affect the observed colors (only solar-scaled abundance ratio models are considered here). To assess whether abundance ratios could compensate for the extra steepness in the data-model difference, we also plot in Figure 5 a green vector which represents the effect of a super-solar abundance ratio star at a given $\mathrm{T}_{\text {eff }} / \log (\mathrm{g}) / Z$ from the synthetic stellar models of Coelho et al. (2005) (see the figure caption for details). The Coelho models do not extend far enough into the NIR for an accurate $K$-band estimate. A combined effect of both TP-AGB treatment and super-solar $[\alpha / \mathrm{Fe}]$ would be represented by the addition of the two vectors and resulting in a steepening that more closely accounts for the data/model offsets.

As a further check on the model predictions, we plot in Figure 6 NIR-NIR colors for both galaxies. Again we see a difference between the observations and model predictions, but not in the same sense as the optical-NIR colors. While the $J-H$ color is predicted to be redder in the Mar05 models, the $J-K$ and $H-K$ colors are predicted to be bluer. As for the optical-NIR colors, the Mar05 models could account for these differences. The only mismatch is in the $H-K$ versus $J-H$ colors for UGC 2124, but this could very well be another manifes-

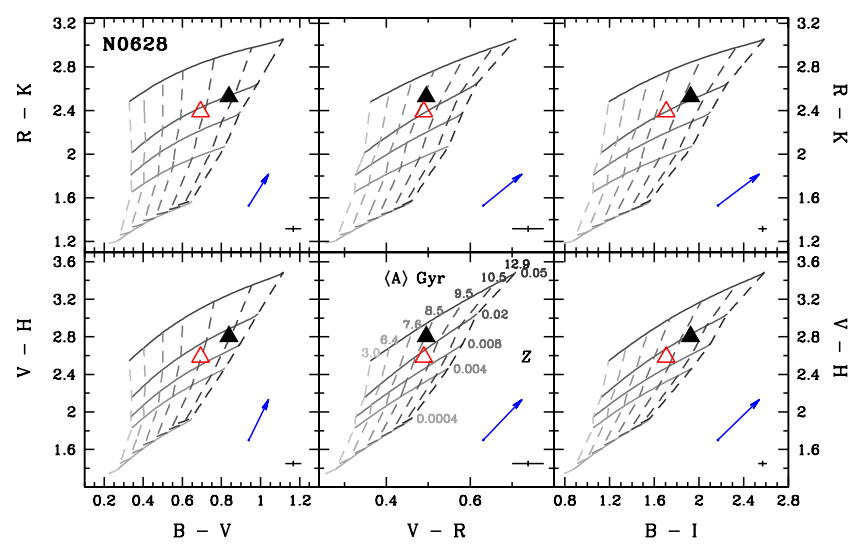

FIG. 4.- Color-color plots for NGC 628. The red open triangles are colors measured ("predicted") from the BC03 model full population synthesis fits to the observed central spectrum. The black solid triangles are the corresponding observed colors. Observational errors (calibration and sky subtraction) are indicated at lower right. Overplotted are grids of BC03 models with exponential SFHs (see MacArthur et al. 2004 for details). Solid and dashed lines are iso- $Z$ and iso-age, respectively, with darker shading indicating higher $Z \mathrm{~s}$ and older ages; see middle bottom panels for labels of average age and $Z$. Blue arrows: model prediction differences between the $1 \mathrm{Gyr} / Z=0.05$ BC03 SSP (red dashed curve in Figure 3) and the Mar05 $1 \mathrm{Gyr} / Z=0.04 \mathrm{SSP}$ (solid blue curve in Figure 31). The arrows point toward the Mar05 predictions (from their respective location for the BC03 models).

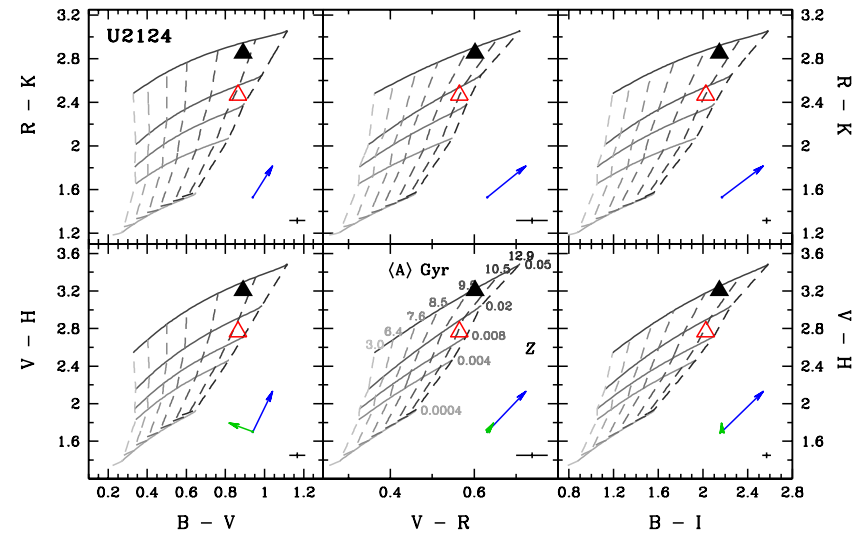

Fig. 5.- Same as Figure 4, but for UGC 2124 and the green arrows show the effect of a non-solar abundance ratio SSP on the colors. Specifically, this represents the difference between $[\alpha / \mathrm{Fe}]=0.4$ and $[\alpha / \mathrm{Fe}]=0$ stars with $\mathrm{T}_{\text {eff }}=4000 \mathrm{~K}$, $[\mathrm{Fe} / \mathrm{H}]=$ solar, $\log (g)=3.0$ from the models of Coelho et al. (2005).

tation of $[\alpha / \mathrm{Fe}]$-enhanced SPs (whose predictions do not extend far enough to test this).

\section{DISCUSSION}

The above analysis has important implications for the modeling and interpretations of integrated stellar populations. The full population synthesis fitting of NGC 628 and UGC 2124 indicates the presence of a young component $(\sim 1 \mathrm{Gyr}$, where Mar05 and BC03 model predictions differ significantly) contributing $\sim 50 \%$ to the $V$-band flux. In SPS models which account for a detailed 

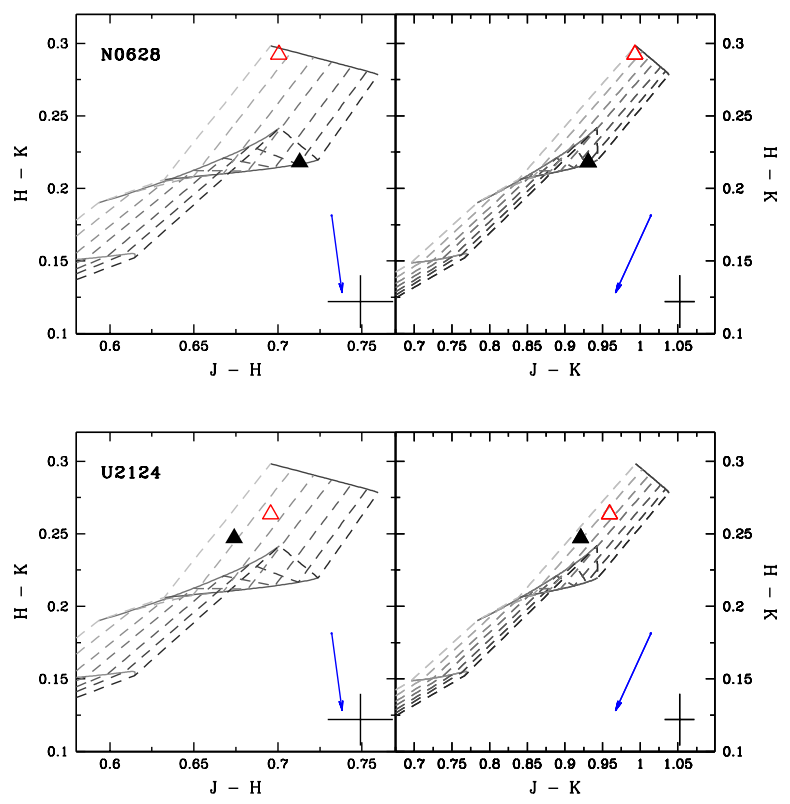

FIG. 6.- Same as in Figure 4 but for NIR colors.

treatment of all stages of the TP-AGB stellar evolution phase, this young population would contribute a significant amount of NIR flux, resulting in very red opticalNIR colors, a mild reddening in $J-H$, and a bluing of the $H-K$ and $J-K$ colors. All of these predicted trends were confirmed with the observed colors, thus providing further support for the presence of the young component, in addition to strongly favoring the contribution of the TP-AGB phase used in the Mar05 SPS models (as well as the Marigo \& Girardi (2007) models which agree well with the Mar05 predictions).

Such anomalous colors have been reported before. For a sample of 5800 galaxies from the SDSS, Eminian et al. (2008) compared optical and NIR colors with quantities derived from spectra (star formation rate (SFR), age, $Z$, and dust attenuation). They found that galaxies with higher SFRs, while having bluer optical colors $(g-r)$, also had redder NIR colors $(H-K)$, which they interpreted as being in qualitative agreement with the dominance in NIR light of the TP-AGB phase after a burst of SF.
At higher redshift, Ellis, Abraham, \& Dickinson (2001) observed the colors of bulges out to $z \sim 1$ and found that, while many of the bulges had blue optical colors, consistent with recent $\mathrm{SF}$, at $z \gtrsim 0.5$, the same bulges had red observed $J-H$ colors. At these higher redshifts, this translates roughly into rest-frame $i-J$. Indeed, while the Mar05 models only show a mild reddening compared to those of BC03 of the $J-H$ color (by $\sim 0.1 \mathrm{mag}$ for a solar 1 Gyr SSP), the $i-J$ color is much redder (by $\sim 0.7 \mathrm{mag}$ ), thus explaining why the effect is only observed at higher$z$. While Ellis et al. attributed the red NIR colors to a predominantly "short burst" mode of bulge building at high- $z$, the red NIR colors could also be explained by the presence of young SPs in the active TP-AGB phase.

These results indicate that caution must be taken when interpreting the optical-NIR colors of integrated stellar populations. Red colors have typically been interpreted as being due to some combination of old age, high $Z$, and significant dust extinction. However, we have shown here that some of the redness could actually be due to a significant contribution to the SED from a young SP with significant NIR flux excess from the TP-AGB evolutionary phase. Further confirmation of this result and its implications awaits larger samples and analysis with forthcoming higher-resolution implementations of the SP models which include proper treatment of the TP-AGB stellar evolutionary phase.

We thank Claudia Maraston for stimulating discussions. LAM acknowledges financial support from the National Science and Engineering Council of Canada (NSERC). SC acknowledges financial support through a Discover Grant from the NSERC. This study is based on observations obtained at the Gemini Observatory, which is operated by the Association of Universities for Research in Astronomy, Inc., under a cooperative agreement with the NSF on behalf of the Gemini partnership: the National Science Foundation (United States), the Science and Technology Facilities Council (United Kingdom), the National Research Council (Canada), CONICYT (Chile), the Australian Research Council (Australia), Ministŕio da Ciência e Tecnologia (Brazil), and Ministerio de Ciencia, Tecnología e Innovación Productiva (Argentina).

\section{REFERENCES}

Bruzual, A. G. \& Charlot, S. 2003, MNRAS, 344, 1000 (BC03)

Carollo, C. M., Scarlata, C., Stiavelli, M., Wyse, R. F. G., \& Mayer, L. 2007, ApJ, 658, 960

Chabrier, G. 2003, PASP, 115, 763

Charlot, S. \& Fall, S. M. 2000, ApJ, 539, $718(\mathrm{C}=\mathrm{F} 00)$

Cid Fernandes, R., Mateus, A., Sodré, L., Stasińska, G., \& Gomes, J. M. 2005, MNRAS, 358, 363

Coelho, P., Barbuy, B., Meléndez, J., Schiavon, R. P., \& Castilho, B. V. 2005, A\&A, 443, 735

Coelho, P., Bruzual, G., Charlot, S., Weiss, A., Barbuy, B., \& Ferguson, J. W. 2007, MNRAS, 382, 498

Courteau, S. 1996, ApJS, 103, 363

Ellis, R. S., Abraham, R. G., \& Dickinson, M. 2001, ApJ, 551, 11 Eminian, C., Kauffmann, G., Charlot, S., Wild, V., Bruzual, G., Rettura, A., \& Loveday, J. 2008, MNRAS, 384, 930

Heavens, A. F., Jimenez, R., \& Lahav, O. 2000, MNRAS, 317, 965

Hook, I. M., Jørgensen, I., Allington-Smith, J. R., Davies, R. L., Metcalfe, N., Murowinski, R. G., \& Crampton, D. 2004, PASP, 116,425 [GMOS]

Landolt, A. U. 1992, AJ, 104, 340
Le Borgne, D., Rocca-Volmerange, B., Prugniel, P., Lançon, A., Fioc, M., \& Soubiran, C. 2004, A\&A, 425, 881

Lee, H.-c., et al. 2009, ApJ, 694, 902

MacArthur, L. A., Courteau, S., Bell, E., \& Holtzman, J. A. 2004, ApJS, 152, 175

MacArthur, L. A., Courteau, S., \& Holtzman, J. A. 2003, ApJ, 582,689

MacArthur, L. A., González, J. J., \& Courteau, S. 2009, MNRAS, 395, 28 [Mac09]

Maraston, C. 2005, MNRAS, 362, 799 [Mar05]

Maraston, C., Daddi, E., Renzini, A., Cimatti, A., Dickinson, M., Papovich, C., Pasquali, A., \& Pirzkal, N. 2006, ApJ, 652, 85

Marigo, P., \& Girardi, L. 2007, A\&A, 469, 239

Peletier, R. F., Balcells, M., Davies, R. L., Andredakis, Y.

Vazdekis, A., Burkert, A., \& Prada, F. 1999, MNRAS, 310, 703

Schiavon, R. P. 2007, ApJS, 171, 146

Schlegel, D. J., Finkbeiner, D. P., \& Davis, M. 1998, ApJ, 500 525

Skrutskie, M. F., et al. 2006, AJ, 131, 1163 [2MASS] 
Walcher, C. J., Böker, T., Charlot, S., Ho, L. C., Rix, H.-W. Rossa, J., Shields, J. C., \& van der Marel, R. P. 2006, ApJ, 649,692 